\title{
Handwriting Movements of Chinese Children with Autism Spectrum Disorder: A Digitized Analysis Using Arabic Numeral Writing Assignments
}

\author{
Tingting Ren ${ }^{1,2}$, Fang $\mathrm{Li}^{1}$, Wenjing $\mathrm{Li}^{1,2}$, Jianfei Luo ${ }^{1}$, and Zhongcheng $\mathrm{Wu}^{1+}$ \\ ${ }^{1}$ High Magnetic Field Laboratory, Chinese Academy of Sciences, Hefei, China \\ ${ }^{2}$ University of Science and Technology of China, Hefei, China
}

\begin{abstract}
The study analyzed handwriting characteristics of Chinese autism spectrum disorders (ASD) children using Arabic numeral handwriting tasks. 10 children with ASD and 11 matched typical developing (TD) subjects were required to write Arabic numeral sequence from 0 to 9 and write the numeral 3 repetitively. Measures in spatial, kinematic and dynamic aspects were extracted. Besides, dynamic time warping (DTW) algorithm was explored to analyze the shape feature in repetitive writing task. The results indicated that the ASD children wrote with significantly larger size, higher velocity and higher acceleration in the Arabic numeral sequence task. In repetitive writing of the numeral 3, the ability in keeping similar number shapes was significantly worse for ASD children, as measured by the DTW distance. The results suggest that the designed Arabic numeral handwriting tasks are effective in evaluating handwriting skills of ASD, which would offer a reference for further research and thus contribute to targeted intervention in ASD.
\end{abstract}

Keywords: handwriting, autism spectrum disorders, motor difficulties, dynamic time warping

\section{Introduction}

As one of the most important tools for communication and expression, handwriting ability has been wildly analyzed to study the motor control mechanisms of fine motor skills $[1,2]$ and the underlying pathology of different diagnostic groups $[3,4]$. As for autism spectrum disorders (ASD), poor handwriting performance is a representative affected motor ability that has been observed in this population [5].

Traditional study of handwriting in ASD was restricted to observational product-oriented tests using various handwriting evaluation scales [6]. As the development of digital tablet technology, analysis of the underlying handwriting process has become possible. In the recent study, Beth P. Johnson et al. [7, 8] measured handwriting features of Australia children by means of assessing cursive writing of four letter ' 1 '. Rosenblum, Simhon, and Gal [9] assessed Hebrew samples of three graded writing tasks, name writing, paragraph copying, and free-style writing, from children with and without high-functioning autism spectrum disorder (HFASD).

Reviewing the literatures on ASD, the writing assignments were language based with a large portion using a Latin-based character set. This would pose considerable barriers when providing a reference for countries outside the Latin language system. Although an increasing attention has gained from researchers, handwriting analysis is still facing the problems in developing an universal assessment method. As for China, reliable and informative study of handwriting process analysis in ASD has not yet been conducted. As far as we know, the present study is the first to explore the characteristic of handwriting in Chinese ASD children.

In our research, to reduce the influence of different language systems, we introduced Arabic numeral handwriting tasks with the expectation that Arabic numeral handwriting tasks would be an alternative to

\footnotetext{
+ Corresponding author. Tel.: +86 055165595140; fax: +86 055165595140.
}

E-mail address: zcwu@iim.ac.cn. 
word handwriting tasks. The goal of this study was to examine the handwriting performance of Chinese ASD children. Spatial, kinematic and dynamic measures were extracted. Moreover, a novel technology, namely dynamic time warping, was introduced for analysis samples of repetitive writing of single Arabic number, which would supply extra information of trajectory formation.

\section{Methods}

\subsection{Participants}

Totally, 21 children between 7 and 9 years of age participated in the research: 10 children with ASD ( 9 males, 1 female, mean age 8.72 years, $\mathrm{SD}=0.99$ years) and 11 TD subjects ( 10 males, 1 female, mean age 9.06 years, $\mathrm{SD}=1.05$ years). All participates were born in China, used Chinese as their first language, and were right-hand dominant.

The ASD children were diagnosed by professional doctors in Chinese public hospital. In addition, the Autism Behavior Checklist (ABC) [10] was performed by the parents before the experiment. None of the ASD individual had a discrepancy between the clinic diagnosis and the $\mathrm{ABC}$ results. The TD group was firstly selected based on the results of ABC. Besides, the TD group had no records of neurological or developmental disorders based on the review of the parents and the teachers.

\subsection{Instruments and Procedure}

The experiment was based on a computer-controlled digital tablet (Wacom, Intuos Pro) and an electronic ink pen (Wacom ink pen). The writing paper was fixed on the surface of the tablet. The handwriting data including pen-tip $\mathrm{X}$-axis position, pen-tip Y-axis position and writing pressure were acquired at a sampling rate of $200 \mathrm{~Hz}$, and the spatial accuracy was $0.01 \mathrm{~mm}$. The children were asked to write with their accustomed manners. Handwriting tasks were described as follows:

1) Writing of Arabic numeral sequence: write the Arabic numeral from 0 to 9 on the paper fixed on the tablet. The subjects were required to write from left to right on the blank area of the paper.

2) Repetitive writing of the Arabic numeral 3: write the Arabic numeral 3 six times inside the 6 rectangles pre-printed on the sheet of the paper.

Fig. 1 shows examples written by the TD and ASD subjects.
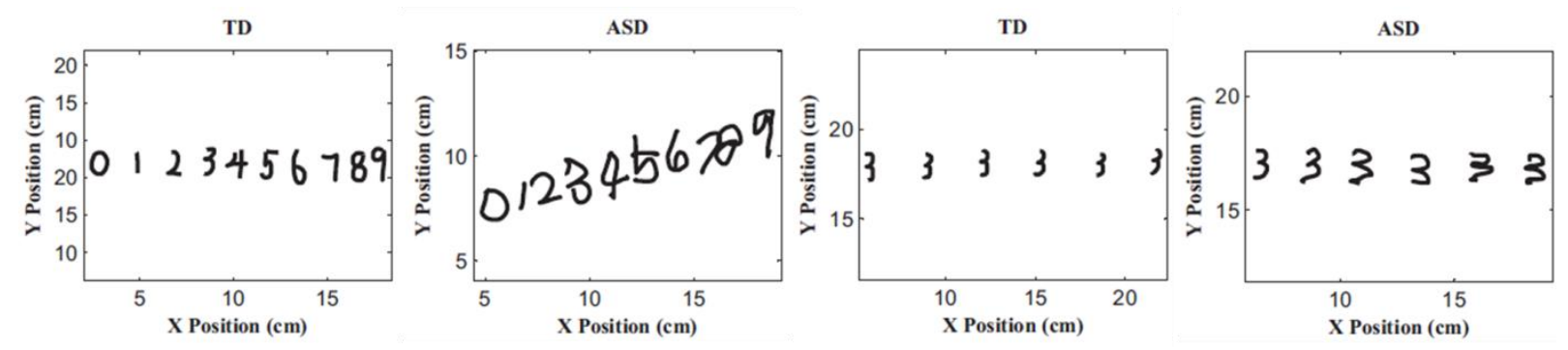

Fig. 1: Handwriting examples written by the TD and ASD subjects.

\subsection{Outcome Measures and Statistical Analysis}

We extracted measures in three categories: spatial measures, kinematic measures and dynamic measures. The spatial measures include the accumulation of trajectory length (ATL), the height of the number, the width of the number and the size of the number. As for the kinematic measures, mean and standard deviation of stroke velocity and acceleration were extracted. Mean and standard deviation of stroke axial pen pressure were assessed to reflect dynamic aspects of handwriting movement. The axial pen pressure was measured in non-scaled units from 0 to 1024. In the present study, a stroke was defined as the sequential written line from the point at which the pen touches the paper until the point it leaves the paper.

In addition, for repetitive writing of the numeral 3, the DTW distance that derived from DTW algorithm was used to measure similarity of two writing shapes. For each participant, the DTW algorithm was carried out between each two of the 6 trials, which means $\mathrm{C}_{6}^{2}$ times of DTW algorithm were conducted. We refer the interested readers to reference ${ }^{[11]}$ for a detailed review of DTW. 
A series of T-test was applied to analyze the differences between ASD and TD children in handwriting measures.

\section{Results}

Table 1 presents an overview of the mean value of the variables which indicate significant differences between groups when performing the Arabic numeral sequence writing task.

The accumulated trajectory length showed great group difference, with significant longer trajectory in the ASD group. Corresponding with this outcome, the average width and size of the number in ASD children were significantly larger than TD children. A detailed comparison was displayed in Fig. 2.

Table 1: A Comparison of Handwriting Variables in Arabic Numeral Sequence Writing Task between ASD and TD Groups

\begin{tabular}{llll}
\hline Measures & ASD & TD & P \\
\hline ATL $(\mathrm{cm})$ & $31.22 \pm 9.03$ & $19.97 \pm 3.01$ & 0.016 \\
Width $(\mathrm{cm})$ & $1.38 \pm 0.43$ & $0.83 \pm 0.16$ & 0.013 \\
Height $(\mathrm{cm})$ & $2.44 \pm 0.84$ & $1.76 \pm 0.33$ & 0.0081 \\
Size $\left(\mathrm{cm}^{2}\right)$ & $3.69 \pm 2.18$ & $1.50 \pm 0.51$ & 0.038 \\
Velocity $(\mathrm{cm} / \mathrm{s})$ & $8.80 \pm 2.62$ & $5.40 \pm 2.26$ & 0.018 \\
SD Velocity $(\mathrm{cm} / \mathrm{s})$ & $2.24 \pm 0.58$ & $1.24 \pm 0.40$ & 0.002 \\
Acc $\left(\mathrm{cm} / \mathrm{s}^{2}\right)$ & $1.45 \mathrm{E}-4 \pm 3.59 \mathrm{E}-5$ & $8.02 \mathrm{E}-5 \pm 3.39 \mathrm{E}-5$ & 0.003 \\
SD Acc $\left(\mathrm{cm} / \mathrm{s}^{2}\right)$ & $6.44 \mathrm{E}-5 \pm 5.65 \mathrm{E}-6$ & $3.79 \mathrm{E}-5 \pm 2.45 \mathrm{E}-5$ & 0.016 \\
\hline
\end{tabular}

Note: Acc, Acceleration. ATL, Accumulated Trajectory Length.

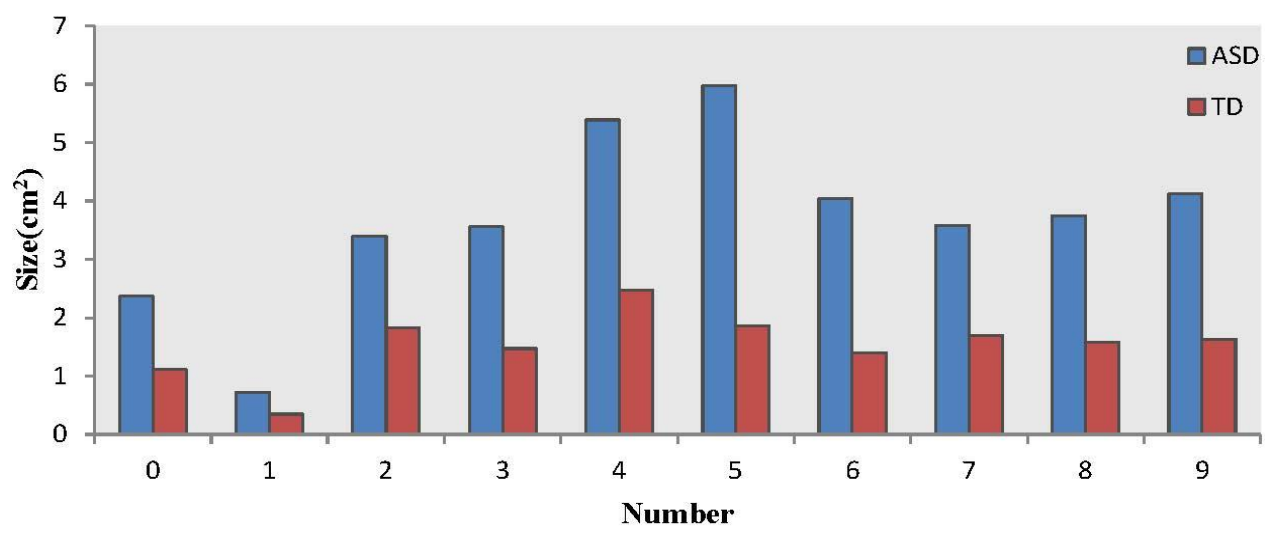

Fig. 2: Comparison of the size of each number (0 to 9) between the ASD and the TD groups.

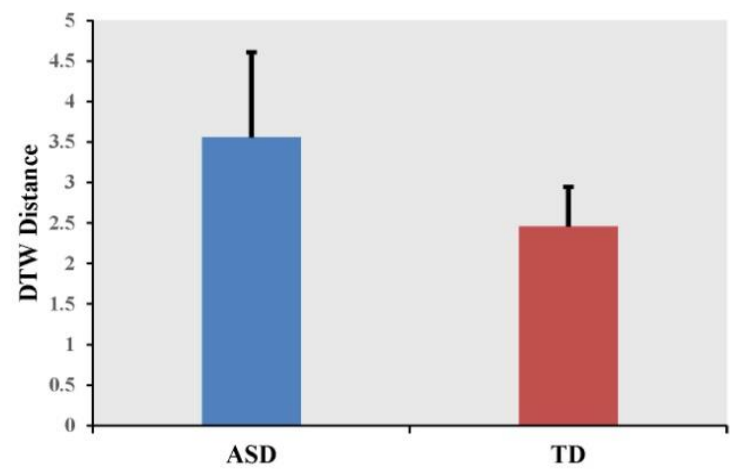

Fig. 3: Comparison of the DTW distance between the ASD and the TD groups.

Significant higher value of mean velocity and acceleration were found in ASD subjects. The SD of velocity and the SD of acceleration were significant greater in ASD group as well. There were no statistically significant differences between groups in mean axial pressure and the SD of axial pressure. 
As to the task of repetitive writing of the numeral 3, although the spatial measures had a slight increase in ASD children, the difference did not reach statistically significant level. The SD of velocity was significantly higher in ASD participants(1.13 and 0.45 in ASD and TD group respectively, $\mathrm{p}=0.001$ ). The dynamic measures in repetitive writing of numeral 3 showed no statistically significant differences between groups.

The mean DTW distance in repetitive writing of the number 3 for the two groups are shown in Fig. 3. Statistic analysis indicated great difference between groups $(\mathrm{p}=0.02)$.

\section{Discussion}

This study is the first to analyze handwriting movement of ASD children in China. Based on the Arabic numeral writing tasks, measures regarding to spatial, kinematic and dynamic aspects were used to compare the handwriting performance of ASD children with the TD controls.

In the Arabic numeral sequence writing task, the accumulation of trajectory length (ATL), width of the overall writing trajectory and the size of the overall writing trajectory were significant higher in ASD children. The combination of these features indicated the finding of writing larger among children with ASD. Nevertheless, there were no significant differences in the writing width, height and size between groups in the repetitive writing of the numeral 3.

The size of the writing products were commonly compared between the ASD and the control groups. The finding of writing large was supported by previous studies on ASD children and adults $[5,7,9,12]$. While the divergence between Arabic numeral sequence task (writing from 0 to 9) and the repetitive numeral writing task may be caused by the differences of the two types of tasks. Firstly, the rectangles used in the repetitive numeral writing task may help ASD children control writing size. Secondly, the Arabic numeral sequence writing task involved more complex changes of letter formation during the process of writing from 0 to 9 .

In the Arabic numerals writing task, the ASD group was characterized by significant higher mean velocity and acceleration. This result was supported by the previous findings of Johnson, Papadopoulos, et al. [7] and Johnson, Phillips, et al. [8], while differed with others which suggest a slowness [9, 13] or comparable speed [14] of handwriting among children with ASD. Nevertheless, there were no group differences of mean velocity and acceleration in repetitive numeral writing task. Further attention should be paid to explore the factors that may impact the writing speed and acceleration of ASD.

The current result on DTW distance was in line with our expectation. In a previous study of letter-form analysis using DTW, poor handwriting was characterized by much higher variability in the letter forms [15]. In our research, when writing the numeral 3 six times, the DTW distance was significant higher in ASD subjects, which indicated that the ASD children were more variable in producing similar number shapes. The ability in keeping similar letter shapes between repetitions of the same handwriting assignment may be impaired for ASD children.

The axial pressure of handwriting in ASD was rarely reported in previous studies. The present finding as to the mean axial pressure was conflict with a previous exploration by which reported a significantly lower value of axial force in ASD group ${ }^{[16]}$. Whereas, our results revealed a similar performance in axial force of children with ASD when compared to TD controls. One possible explanation for the discrepancy may relate to the type of writing. The continuous circles drawing task requires sustaining control of axial force exerted on the paper from the start to the end of writing movement. In the present study, the Arabic numerals are separated and consist of several strokes, which bring about pen lifts for subjects to get a relief during the course of handwriting. Children with ASD may be more easily to feel tired in the continuous task.

This paper has comprehensively examined the handwriting feature using numerals writing tasks in Chinese ASD children for the first time. The results indicated that the designed simple Arabic numeral handwriting tasks are effective in evaluating handwriting skills of individuals with ASD, which would offer a reference for further research. The findings leave a cue for clinicians and researchers who would like to get the underlying mechanism in ASD, and for therapist and educators who would like to optimize target rehabilitation as well as proper education to improve the motor and handwriting ability in ASD children. 


\section{Acknowledgements}

We are grateful to the children who participate in this study. This study is supported by grants from the National Natural Science Foundation of China (No. 61301058) and Natural Science Foundation of Anhui Province (No. 1408085QF109).

\section{References}

[1] Lin, Q., et al., Characterization of fine motor development: Dynamic analysis of children's drawing movements. Human Movement Science, 2015. 40: p. 163-175.

[2] Smits-Engelsman, B., et al., Fine motor deficiencies in children with developmental coordination disorder and learning disabilities: An underlying open-loop control deficit. Human Movement Science, 2003. 22(4): p. 495-513.

[3] Prunty, M.M., et al., Handwriting speed in children with Developmental Coordination Disorder: Are they really slower? Research in Developmental Disabilities, 2013. 34(9): p. 2927-2936.

[4] Shen, I.H., T.Y. Lee, and C.L. Chen, Handwriting performance and underlying factors in children with Attention Deficit Hyperactivity Disorder. Res Dev Disabil, 2012. 33(4): p. 1301-9.

[5] Frith, U., Autism and Asperger syndrome. 1991: Cambridge University Press.

[6] Kushki, A., T. Chau, and E. Anagnostou, Handwriting difficulties in children with autism spectrum disorders: A scoping review. Journal of autism and developmental disorders, 2011. 41(12): p. 1706-1716.

[7] Johnson, B.P., et al., A quantitative comparison of handwriting in children with high-functioning autism and attention deficit hyperactivity disorder. Research in Autism Spectrum Disorders, 2013. 7(12): p. 1638-1646.

[8] Johnson, B.P., et al., Understanding macrographia in children with autism spectrum disorders. Research in developmental disabilities, 2013. 34(9): p. 2917-2926.

[9] Rosenblum, S., H.A.B. Simhon, and E. Gal, Unique handwriting performance characteristics of children with high-functioning autism spectrum disorder. Research in Autism Spectrum Disorders, 2015.

[10] Krug, D.A., J. Arick, and P. Almond, Behavior checklist for identifying severely handicapped individuals with high-levels of autistic behavior. Journal of Child Psychology and Psychiatry and Allied Disciplines, 1980. 21(3): p. 221-229.

[11] Sakoe, H. and S. Chiba, Dynamic programming algorithm optimization for spoken word recognition. Acoustics, Speech and Signal Processing, IEEE Transactions on, 1978. 26(1): p. 43-49.

[12] Beversdorf, D.Q., et al., Brief report: macrographia in high-functioning adults with autism spectrum disorder. Journal of autism and developmental disorders, 2001. 31(1): p. 97-101.

[13] Hellinckx, T., H. Roeyers, and H. Van Waelvelde, Predictors of handwriting in children with autism spectrum disorder. Research in Autism Spectrum Disorders, 2013. 7(1): p. 176-186.

[14] Cartmill, L., S. Rodger, and J. Ziviani, Handwriting of eight-year-old children with autistic spectrum disorder: An exploration. Journal of Occupational Therapy, Schools, \& Early Intervention, 2009. 2(2): p. 103-118.

[15] Di Brina, C., et al., Dynamic time warping: A new method in the study of poor handwriting. Human movement science, 2008. 27(2): p. 242-255.

[16] Fleury, A., et al., Statistical persistence and timing characteristics of repetitive circle drawing in children with ASD. Developmental neurorehabilitation, 2013. 16(4): p. 245-254. 